\title{
DISTRIBUIÇÃO DE MERCÚRIO NA FAUNA ESTUARINA DO RIO JAGUARIBE - CE
}

\author{
Mercury distribution in the estuarine fauna \\ of the Jaguaribe river - CE
}

\author{
Victor Lacerda Moura ${ }^{1 *}$, Breno Gustavo Bezerra Costa², Luiz Drude de Lacerda ${ }^{3}$ \\ ${ }^{1}$ Doutorando do Instituto de Ciências do Mar, Universidade Federal do Ceará, Bolsista da Fundação Cearense de Apoio \\ ao Desenolvimento Científico e Tecnológico (FUNCAP), Av. Abolição 3207, Fortaleza, CE 60165-081. \\ E-mail: victuh00@gmail.com \\ 2 Professor do Instituto Socioambiental e dos Recursos Hídricos, Universidade Rural da Amazônia, Avenida Presidente \\ Tancredo Neves 2501, Belém, PA 66077-830. E-mail: breno_pesca@hotmail.com \\ ${ }^{3}$ Professor do Instituto de Ciências do Mar, Universidade Federal do Ceará, Av. Abolição 3207, Fortaleza, CE 60165-081. \\ E-mail: Idrude@pq.cnpq.br \\ *Autor para correspodência
}

\begin{abstract}
RESUMO
Modificações na paisagem natural aliadas às mudanças climáticas globais, alteram a hidrodinâmica da Bacia Inferior do rio Jaguaribe, possibilitando maior biodisponibilidade de contaminantes, entre eles o mercúrio $(\mathrm{Hg})$. Análise das concentrações de $\mathrm{Hg}$ na fauna estuarina (13 espécies de peixes e 5 de invertebrados) mostraram variações nas concentrações de Hg relacionadas aos hábitos alimentares das espécies. Tanto entre invertebrados quanto nos peixes, as maiores concentrações foram encontradas nas espécies carnívoras. Entretanto, não foram encontradas diferenças significativas entre as concentrações médias entre peixes carnívoros e onívoros, e entre invertebrados carnívoros e filtradores, o que pode estar relacionado as características biológicas específicas, como tamanho, estado de maturação e composição da dieta.
\end{abstract}

Palavras-chave: Estuário. Mercúrio. Bioacumulação. Hábito alimentar.

\begin{abstract}
Modifications in the natural landscape of the Lower Jaguaribe River Basin, associated with global climate changes, contribute to changes in the hydrodynamics of the Lower Basin of Jaguaribe
\end{abstract}

Recebido em: 04/07/2018

Aprovado em: 23/07/2018

Publicado online em: 05/09/2018 
riverecosystem, increasing contaminants bioavailability, among them mercury ( $\mathrm{Hg})$. Concentrations of $\mathrm{Hg}$ measured in the estuarine fauna (13 species of bonyfish and 5 of invertebrates) showed variations in $\mathrm{Hg}$ concentrations related to feeding habits. Highest concentrations in both, invertebrates and fish, were found in carnivorous species. However, no significant differences were found between the mean $\mathrm{Hg}$ concentrations in carnivores and omnivores fishes, and between carnivorous and filter feeders invertebrates, which may be related to intrinsic characteristics such as size, sexual maturity and diet composition.

Keywords: Estuary. Mercury. Bioaccumulation. Feeding Habit.

\section{INTRODUÇÃO}

O mercúrio (Hg) possui ampla distribuição global, podendo ser encontrado no ambiente sob diferentes espécies químicas (Streets et al., 2017). A especiação química do Hg é bastante diversa, podendo ocorrer ligado a compostos orgânicos e inorgânicos. Entretanto, a forma orgânica (metilmercúrio $-\mathrm{CH}_{3} \mathrm{Hg}^{+}$) é considerada a espécie de maior preocupação ambiental, devido a sua elevada toxicidade e seu alto tempo de residência nos organismos, que resulta em elevadas taxas de bioacumulação e biomagnificação (Beckers \& Rinklebe, 2017).

O rio Jaguaribe possui uma zona estuarina impactada pela escassez de chuvas, devido as mudanças climáticas globais, e por modificações na paisagem natural, como a construção de barramentos, que contribuem com alterações na hidrodinâmica e na hidroquímica desse estuário, favorecendo a retenção das massas de água continentais e condições para maior mobilidade e reatividade do $\mathrm{Hg}$, favorecendo sua biodisponibilidade (Lacerda et al., 2013). Estudos recentes (Costa \& Lacerda, 2014; Rios et al., 2016; Moura \& Lacerda, 2018) tem sugerido um aumento na concentração de $\mathrm{Hg}$ na biota local como consequência desta maior biodisponibilidade. Assim, este trabalho objetiva quantificar as concentrações de $\mathrm{Hg}$ na biota da região estuarina do rio Jaguaribe, afim de entender os fatores biológicos e ecológicos responsáveis por sua distribuição.

\section{MATERIAL E MÉTODOS}

As amostras foram coletadas entre 2007 e 2015 com auxílio dos pescadores locais através do uso de artes de pesca típicas, como rede de emalhar e tarrafa, exceto para captura dos moluscos, coletados manualmente. A coleta ocorreu ao longo da zona estuarina do rio Jaguaribe (Figura 1), incluindo espécimes de peixes ósseos, crustáceos (siris e camarões) e moluscos (bivalves e gastrópodes). No campo, as amostras foram acondicionadas e preservadas em recipientes térmicos e encaminhados ao Laboratório de Biogeoquímica Costeira (LABOMAR-UFC), para identificação da espécie e seu hábito alimentar(FISHBASE, 2018), medição e pesagem.

Após identificação, sub-amostras da musculatura, para peixes e crustáceos, e de tecido mole (amostragem composta), para moluscos, foram retiradas e liofilizadas, visando a preservação dessas amostras. Em seguida, o material liofilizado foi tratado, seguindo metodologia descrita por Costa \& Lacerda (2014) para quantificação da concentração de $\mathrm{Hg}$ total nesses organismos. A quantificação do $\mathrm{Hg}$ foi realizada em um espectrômetro de 
absorção atômica com sistema de geração de vapor frio (CVAAS), sendo os resultados expressos em peso seco.

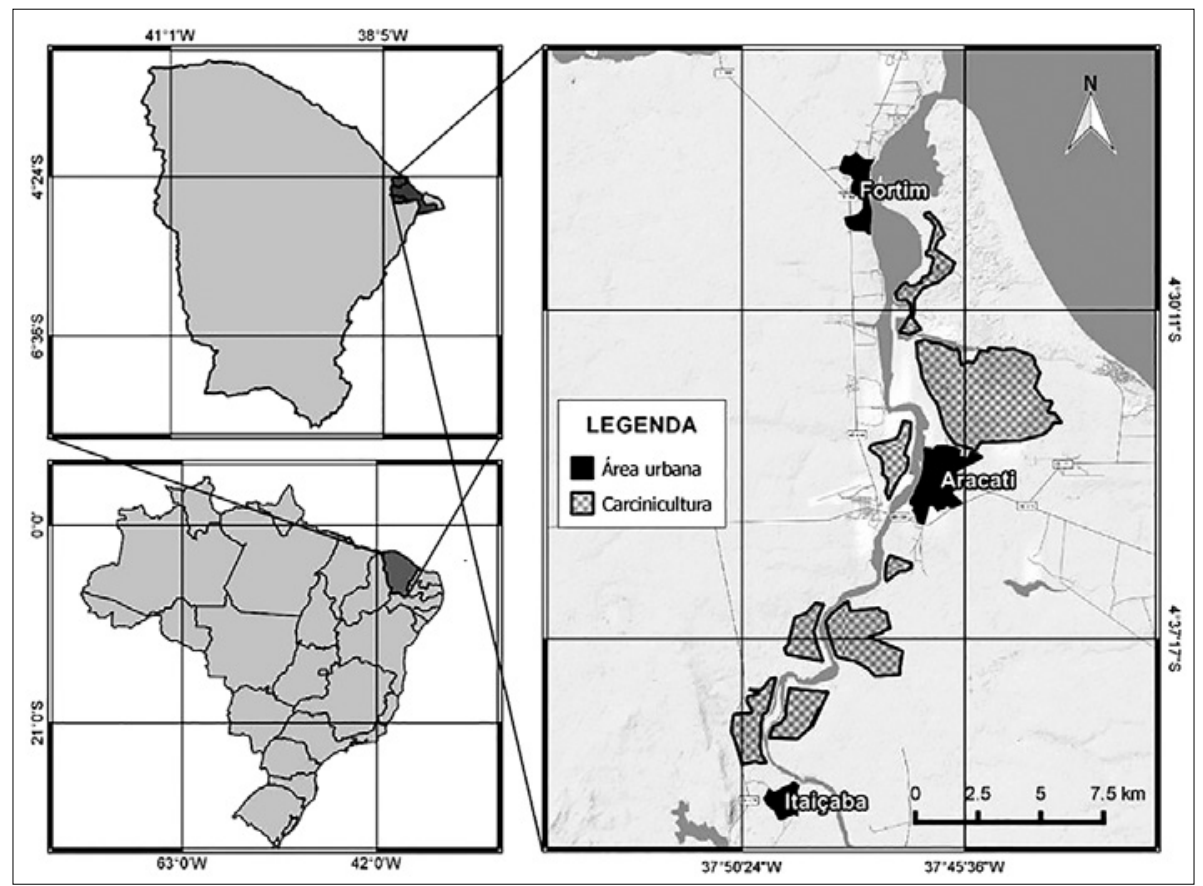

Figura 1 - Localização da zona estuarina do Rio Jaguaribe.

O limite de detecção médio da metodologia utilizada foi de $3 \pm 5 \mathrm{ng}$. $\mathrm{g}^{-1}$, sendo obtido pela média dos resultados do triplo do desvio padrão de sete leituras de brancos multiplicado pelo fator específico determinado pela distribuição $t$ de Student $(3,14$, gl 6) (USEPA, 2000). A validação dos resultados da concentração de $\mathrm{Hg}$ foi obtida por análise, em duplicata, do material de referência certificado CE278K, apresentando recuperação média de 81\%. A normalidade dos dados foi analisada através do teste de Shapiro-Whilk, apresentando resultados para dados não normais. A relação entre as concentrações de Hg e o hábito alimentar apresentado pelas espécies coletadas foi analisada por testes de KruskalWallis e ANOVA, após avaliação da curva dos resíduos. Havendo diferença entre os grupos amostrados, testes a posteriori foram realizados para descobrir quais grupos apresentavam distinção. O valor de significância adotado para os testes foi de $95 \%(p<0,05)$. Os testes estatísticos e os gráficos bloxspot foram realizados no programa Past 3.05 (Copyright Hammer 1999-2015) e Microsoft ${ }^{\circledR}$ Office 2016 (Microsoft Corporation 2016).

\section{RESULTADOS E DISCUSSÃO}

Foram coletados e analisados 188 peixes ósseos e 99 organismos invertebrados, sendo 63 crustáceos e 36 moluscos. A concentração média de Hg total (Tabela I e II) variou entre $12 \pm 4$ e $90 \pm 52$ ng. g $^{-1}$ entre os invertebrados, já entre os peixes variou entre $13 \pm 2$ e $256 \pm$ $198 \mathrm{ng} . \mathrm{g}^{-1}$. As maiores concentrações de $\mathrm{Hg}$ total, entre as espécies de invertebrados, foram observadas em siris do gênero Callinectes (Tabela I), crustáceos carnívoros, que possuem uma dieta composta principalmente por crustáceos e moluscos (Carqueija \& Gôuvea, 1998; Carvalho \& Couto, 2010). As maiores concentrações encontradas podem estar relacionadas 
à elevada bioacumulação e longo tempo de residência do $\mathrm{Hg}$ em espécies carnívoras (Reichmuth et al., 2010).

Tabela I - Número amostral (n), hábito alimentar, comprimento, peso e concentração de Hg nos invertebrados da região estuarina do Rio Jaguaribe, CE.

\begin{tabular}{|c|c|c|c|c|c|}
\hline Espécie & $\mathbf{n}$ & Hábito alimentar & Comp. $(\mathrm{cm})$ & Peso (g) & [Hg] Tot. (ng.g-1) \\
\hline Callinectes sp. & 19 & Carnívoro & $\begin{array}{c}10 \pm 2 \\
(7,5-12,5)\end{array}$ & $\begin{array}{c}77 \pm 43 \\
(30,5-152)\end{array}$ & $\begin{array}{c}90 \pm 53 \\
(21,4-201,4)\end{array}$ \\
\hline Pugilina tupiniquim & 5 & Carnívoro & $\begin{array}{c}9 \pm 1 \\
(9-10)\end{array}$ & $\begin{array}{c}56 \pm 8 \\
(48,6-67,6)\end{array}$ & $\begin{array}{c}46 \pm 12 \\
(32,7-64,7)\end{array}$ \\
\hline Mytella charruana & 10 & Filtrador & N.M.* & $\begin{array}{c}1 \pm 0,4 \\
(0,7-2,2)\end{array}$ & $\begin{array}{c}55 \pm 8 \\
(46-70,3)\end{array}$ \\
\hline Anomalocardia brasiliana & 21 & Filtrador & $\begin{array}{c}2 \pm 0,2 \\
(1,7-2,3)\end{array}$ & $\begin{array}{c}4 \pm 2 \\
(2,5-9,9)\end{array}$ & $\begin{array}{c}52 \pm 13 \\
(27-74,6)\end{array}$ \\
\hline Litopenaeus vannamei & 44 & Detritívoro & $\begin{array}{c}9 \pm 3 \\
(2,9-16) \\
\end{array}$ & $\begin{array}{c}9 \pm 4 \\
(2,5-25,5)\end{array}$ & $\begin{array}{c}13 \pm 6 \\
(7,3-39,5)\end{array}$ \\
\hline
\end{tabular}

* Não foi medido

Foi possível observar uma diferença significativa $(\mathrm{H}=66,12 ; \mathrm{p}<0,05)$ entre as concentrações de $\mathrm{Hg}$ total nos diferentes hábitos alimentares apresentados pelos invertebrados (Figura 2). As concentrações médias de Hg em carnívoros e filtradores foram maiores que nos detritívoros. As menores concentrações encontradas na espécie L. vannamei podem ser devido ao fato desses organismos serem exóticos e provavelmente terem escapado de fazendas de carcinicultura. Concentrações similares de $\mathrm{Hg}$ medidas em L. vannamei, coletados diretamente em tanques de cultivo por Soares et al. (2011) $\left(6,3\right.$ - 15,9 ng.g $\left.{ }^{-1}\right)$ corroboram essa afirmação.

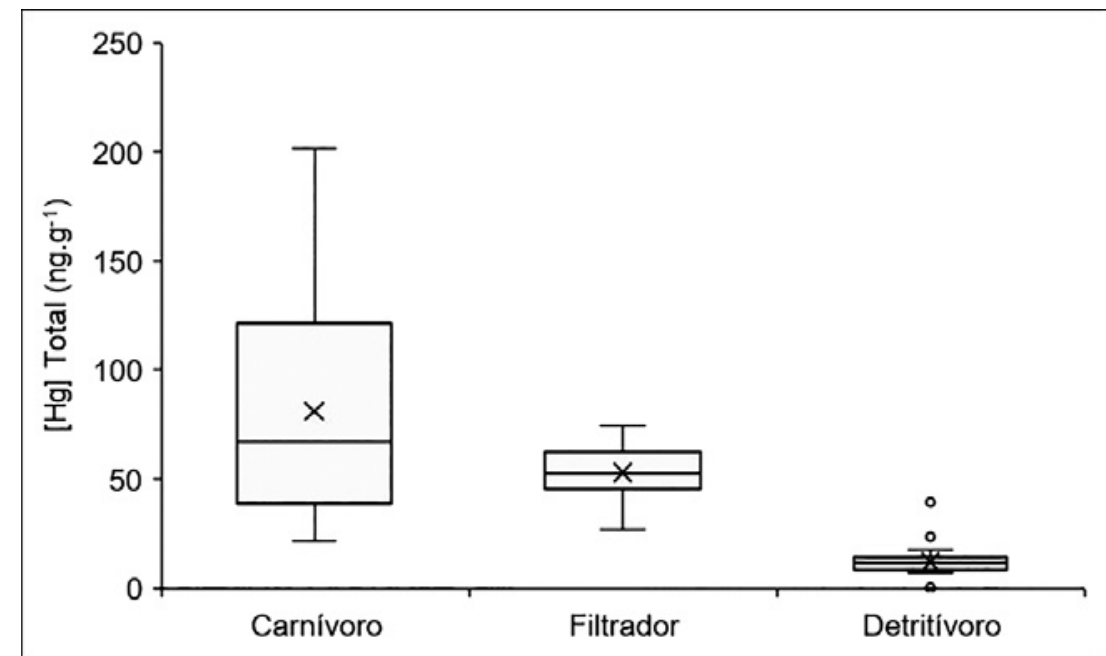

Figura 2 - Relação entre as concentrações de Hg total e o hábito alimentar das espécies de invertebrados da zona estuarina do rio Jaguaribe.

As espécies de invertebrados filtradoras apresentaram concentrações médias similares as espécies carnívoras, inclusive aqueles medidos no gastrópode carnívoro $P$. tupiniquim, apesar de estarem mais próximas a base da cadeia trófica. Este fato pode estar relacionado à assimilação majoritária de espécies químicas dissolvidas na água e adsorvidas em partículas de sedimento ou suspensão por espécies filtradoras, compartimentos mais enriquecidos em espécies de Hg inorgânicas de menor taxa de bioacumulação, em contra- 
posição ao incorporado via dieta, que possui maior concentração de Hg orgânico de bioacumulação mais eficiente (Beckers \& Rinklebe, 2017; Briant et al., 2017).

A maior concentração média de Hg foi observada no ariacó (L. synagris), entre os peixes, espécie de hábito carnívoro, possuindo alimentação composta principalmente por peixes e crustáceos (Allen, 1985). Claramente, os indivíduos desta espécie coletados em 2010 foram significativamente maiores que em 2015, justificando as maiores concentrações de $\mathrm{Hg}$ encontradas em 2010 nesta espécie. Também no robalo (C. parallelus), o maior tamanho em 2010 resultou em maiores concentrações de Hg. Modificações morfológicas e fisiológicas, como o aumento do tamanho da boca, contribuem para aumentar a concentração de Hg entre indivíduos de mesma espécie. Por exemplo, a captura de presas maiores, que geralmente apresentam concentrações de $\mathrm{Hg}$ superiores à de pequenas presas (Chouvelon et al., 2014), ou a captura de um maior número de presas, ocasionam uma maior exposição ao Hg em organismos maiores através da dieta (Dang \& Wang, 2012). Organismos maiores também foram expostos a níveis ambientais do Hg por mais tempo. A espécie $M$. americanus apresentou comportamento oposto, com maiores concentrações de $\mathrm{Hg}$ em indivíduos menores, entretanto o baixo número amostral não permite a discussão deste resultado.

Além de variações intraespecíficas, as concentrações de $\mathrm{Hg}$ também apresentaram variações interespecíficas. Também foram observados, em peixes, maiores concentrações de Hg em espécies carnívoras (Tabela II). Entretanto não houve diferença significativa entre as concentrações médias de carnívoros e onívoros $(U=2710,5 ; p>0,05)$. Por outro lado, as menores concentrações de $\mathrm{Hg}$ em peixes foram observadas em espécies planctívoras (Figura 3).

Tabela II - Número amostral (n), ano de coleta, hábito alimentar, comprimento, peso e concentração de Hg em peixes da região estuarina do Rio Jaguaribe.

\begin{tabular}{|c|c|c|c|c|c|c|}
\hline Espécie & $\mathbf{n}$ & $\begin{array}{l}\text { Ano de } \\
\text { coleta }\end{array}$ & $\begin{array}{c}\text { Hábito } \\
\text { alimentar }\end{array}$ & Comp. (cm) & Peso (g) & [Hg] Tot. (ng.g-1) \\
\hline \multirow{2}{*}{ Lutjanus synagris* } & 4 & 2010 & \multirow[b]{2}{*}{ Carnívoro } & $\begin{array}{c}27 \pm 7 \\
(19-35)\end{array}$ & $\begin{array}{c}325 \pm 222 \\
(100,0-600,0)\end{array}$ & $\begin{array}{c}257 \pm 198 \\
(95,8-545,9)\end{array}$ \\
\hline & 5 & 2015 & & $\begin{array}{c}18 \pm 4 \\
(14-25)\end{array}$ & $\begin{array}{c}85 \pm 55 \\
(39,7-174,6)\end{array}$ & $\begin{array}{c}60 \pm 15 \\
(40,9-80,6)\end{array}$ \\
\hline Plagioscion squamosissimus & 4 & & Carnívoro & $\begin{array}{c}25 \pm 3 \\
(22,5-29)\end{array}$ & $\begin{array}{c}164 \pm 53 \\
(88,0-204,0)\end{array}$ & $\begin{array}{c}141 \pm 85 \\
(51,1-235,1)\end{array}$ \\
\hline Haemulon plumieri & 6 & & Carnívoro & $\begin{array}{c}20 \pm 1 \\
(19-23)\end{array}$ & $\begin{array}{c}133 \pm 26 \\
(100,0-175,0)\end{array}$ & $\begin{array}{c}124 \pm 68 \\
(71,9-250,7)\end{array}$ \\
\hline \multirow{2}{*}{ Centropomus parallelus* } & 11 & 2013 & \multirow{2}{*}{ Carnívoro } & $\begin{array}{c}31 \pm 7 \\
(23-47)\end{array}$ & $\begin{array}{c}285 \pm 159 \\
(140,0-724,0)\end{array}$ & $\begin{array}{c}124 \pm 38 \\
(69,5-202,2)\end{array}$ \\
\hline & 3 & 2015 & & $\begin{array}{c}19 \pm 6 \\
(15-25)\end{array}$ & $\begin{array}{c}58 \pm 51 \\
(24,0-117,5)\end{array}$ & $\begin{array}{c}38 \pm 12 \\
(27,0-50,7)\end{array}$ \\
\hline \multirow{2}{*}{ Menticirrhus americanus* } & 3 & 2013 & \multirow{2}{*}{ Carnívoro } & $\begin{array}{c}24 \pm 1 \\
(22,5-24,5)\end{array}$ & $\begin{array}{c}131 \pm 23 \\
(110,0-156,0)\end{array}$ & $\begin{array}{c}38 \pm 2 \\
(36,3-39,8)\end{array}$ \\
\hline & 6 & 2015 & & $\begin{array}{c}14 \pm 1 \\
(12,5-15)\end{array}$ & $\begin{array}{c}28 \pm 8 \\
(20,1-41,3)\end{array}$ & $\begin{array}{c}103 \pm 30 \\
(64,8-143,7)\end{array}$ \\
\hline Elops saurus & 4 & & Carnívoro & $\begin{array}{c}25 \pm 2 \\
(22,5-27,5)\end{array}$ & $\begin{array}{c}84 \pm 24 \\
(56,7-110,0)\end{array}$ & $\begin{array}{c}98 \pm 29 \\
(57,5-123,7)\end{array}$ \\
\hline Lutijanus cyanopterus & 6 & & Carnívoro & $\begin{array}{c}17 \pm 2 \\
(14,0-19,6)\end{array}$ & $\begin{array}{c}79 \pm 34 \\
(40,0-130,0)\end{array}$ & $\begin{array}{c}96 \pm 23 \\
(76,1-137,3)\end{array}$ \\
\hline Lutjanus jocu & 6 & & Carnívoro & $\begin{array}{c}13 \pm 2 \\
(10-15)\end{array}$ & $\begin{array}{c}32 \pm 10 \\
(18,7-45,8)\end{array}$ & $\begin{array}{c}65 \pm 34 \\
(39,8-128,8)\end{array}$ \\
\hline Archosargus rhomboidalis & 4 & & Onívoro & $\begin{array}{c}27 \pm 3 \\
(24-32)\end{array}$ & $\begin{array}{c}353 \pm 133(260,0- \\
550,0)\end{array}$ & $\begin{array}{c}228 \pm 114(95,1- \\
374,1)\end{array}$ \\
\hline Cathorops spixii & 70 & & Onívoro & $\begin{array}{c}23 \pm 7 \\
(10,8-50)\end{array}$ & $\begin{array}{c}135 \pm 144 \\
(12,0-1774,0)\end{array}$ & $\begin{array}{c}98 \pm 69 \\
(8,7-300,5)\end{array}$ \\
\hline
\end{tabular}




\begin{tabular}{|c|c|c|c|c|c|}
\hline Eugerres brasilianus & 40 & Onívoro & $\begin{array}{c}18 \pm 6 \\
(10,5-27,5)\end{array}$ & $\begin{array}{c}108 \pm 89 \\
(22,0-322,0)\end{array}$ & $\begin{array}{c}79 \pm 81 \\
(17,0-518,1)\end{array}$ \\
\hline Mugil curema & 12 & Planctívoro & $\begin{array}{c}23 \pm 4 \\
(19-33)\end{array}$ & $\begin{array}{c}137 \pm 85 \\
(67,9-286,0)\end{array}$ & $\begin{array}{c}23 \pm 13 \\
(5,4-41,1)\end{array}$ \\
\hline Gobionellus oceanicus & 5 & Planctívoro & $\begin{array}{c}22 \pm 1 \\
(20,5-24,5)\end{array}$ & $\begin{array}{c}32 \pm 7 \\
(23,3-42,4)\end{array}$ & $\begin{array}{c}14 \pm 3 \\
(11,5-17,1)\end{array}$ \\
\hline
\end{tabular}

*Os indivíduos foram separados por ano de coleta devido a diferença significativa entre o comprimento médio para cada período de coleta.

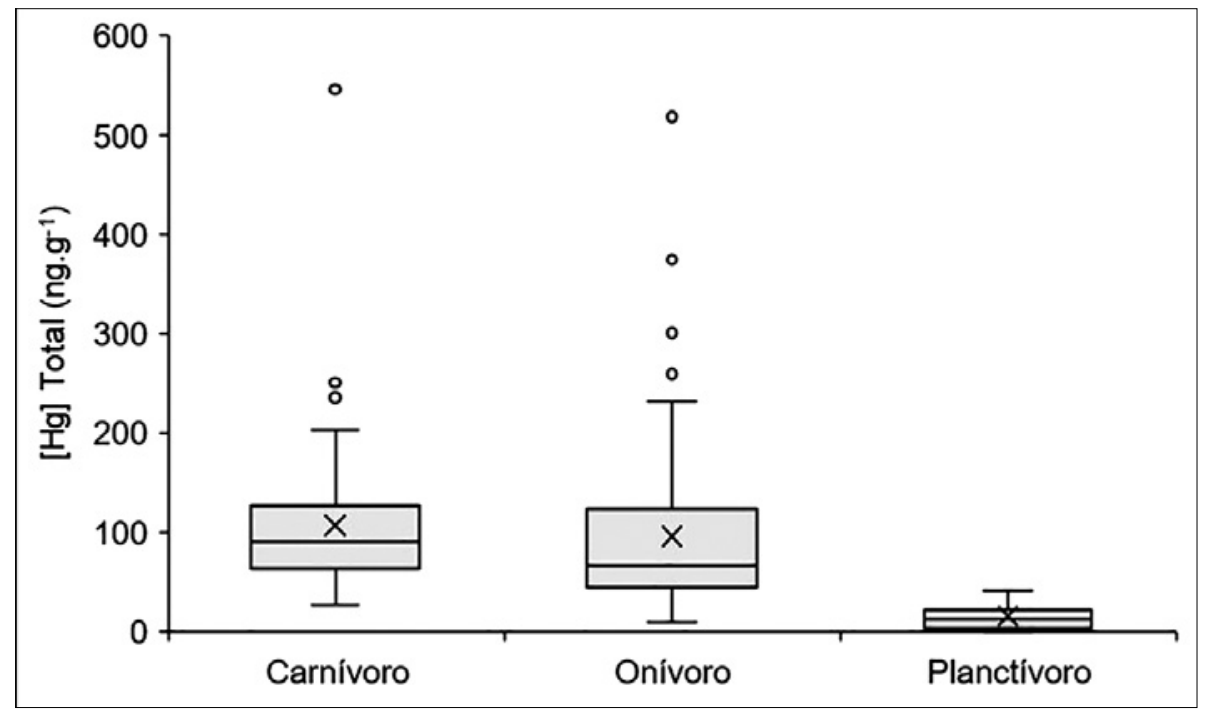

Figura 3 - Relação entre as concentrações de Hg total e o hábito alimentar das espécies de peixes da zona estuarina do rio Jaguaribe.

Soares et al. (2016) também encontraram maiores concentrações de Hg em espécies de peixes carnívoras na bacia do Rio Negro, Amazônia. Pinho et al. (2002), trabalhando com tubarões da plataforma continental, também observou variações na concentração de Hg entre espécies com dietas distintas, com maiores concentrações encontradas em espécies piscívoras. A dieta específica, portanto, contribui para uma maior variância nas concentrações de $\mathrm{Hg}$ entre espécies de hábito alimentar semelhante, demonstrando assim a importância da análise de $\mathrm{Hg}$ em itens de dieta para uma melhor compreensão dos fatores controladores da concentração de $\mathrm{Hg}$ na biota.

As concentrações de $\mathrm{Hg}$ medidas nas espécies de peixes e invertebrados, da região estuarina do rio Jaguaribe estão muito abaixo dos limites estabelecidos pela legislação brasileira vigente (Portaria ${ }^{\circ}$ 685, de 27 de agosto de 1998, da Agência Nacional de Vigilância Sanitária). Entretanto, sugere-se que mudanças nas características hidroquímcias e hidrodinâmicas desse ecossistema estão contribuindo para uma maior biodisponibilidade do $\mathrm{Hg}$, que aliada ao aumento das taxas de consumo pelas comunidades locais, pode tornar-se um risco de exposição humana a longo prazo, apesar das baixas concentrações encontradas, como sugerido em estudos anteriores (Costa \& Lacerda, 2014; Moura \& Lacerda, 2018).

\section{CONCLUSÃO}

Apesar das baixas cargas de $\mathrm{Hg}$ introduzidas na zona estuarina do rio Jaguaribe, suas condições ambientais contribuem para biodisponibilização desse contaminante. 
Contudo, fatores biológicos, como o hábito alimentar e o tamanho, contribuem com variações nas concentrações de Hg entre as espécies e indivíduos, respectivamente, o que pode ser observado tanto nos vertebrados quanto nos invertebrados, resultando em maiores concentrações em espécies carnívoras, quando comparados a espécies próximas a base da cadeia trófica e dentro de uma mesma espécie, em indivíduos maiores.

Agradecimentos - Agradeço a Fundação Cearense de Apoio ao Desenvolvimento Científico e Tecnológico (FUNCAP) e ao Instituto Nacional de Ciência e Pesquisa (INCT) pelo apoio financeiro dado a pesquisa. A toda equipe laboratorial, pelo auxílio nas análises, interpretação e discussão dos dados obtidos.

\section{REFERÊNCIAS BIBLIOGRÁFICAS}

Allen, G. R. Snappers of the world: An annotated and illustrated catalogue of lutjanid species known to date. FAO Fish. Synop., 208p., Rome, 1985.

Beckers, F. \& Rinklebe, J. Cycling of mercury in the environment: Sources, fate, and human health implications: A review. Critical Reviews in Environmental Science and Technology, v. 47, n. 9, p. 693-794, 2017.

Briant, N.; Chouvelon, T.; Martinez, L.; Brach-Papa, C.; Chiffoleau, J. F.; Savoye, N.; Sonke, J. \&Knoery, J. Spatial and temporal distribution of mercury and methylmercury in bivalves from the French coastline. Marine Pollution Bulletin, v. 114, n. 2, p. 1096 - 1102, 2017.

Carqueija, C.R.G. \& Gouvêa, E.P.D. Hábito alimentar de Callinectes larvatus Ordway (Crustacea, Decapoda, Portunidae) no manguezal de Jiribatuba, Baía de Todos os Santos, Bahia. Revista Brasileira de Zoologia, v. 15, n. 1, p. 273-278, 1998.

Carvalho, F.L. \& Couto, E.D.C.G. Dieta do siri Callinectes exasperatus (Decapoda, Portunidae) no estuário do rio Cachoeira, Ilhéus, Bahia. UNICiências, v. 14, n. 2, p. 325-334, 2010.

Chouvelon, T.; Caurant, F.; Cherel, Y.; Simon-Bouhet, B.; Spitz, J. \& Bustamante, P. Species-and size-related patterns in stable isotopes and mercury concentrations in fish help refine marine ecosystem indicators and provide evidence for distinct management units for hake in the Northeast Atlantic. ICES Journal of Marine Science, v. 71, n. 5, p. 1073-1087, 2014.

Costa, B.G.B. \& Lacerda, L.D. Mercury (Hg) in fish consumed by the local population of the Jaguaribe River lower basin, Northeast Brazil. Environmental Science and Pollution Research, v. 21, n. 23, p. 13335-13341, 2014.

Dang, F. \& Wang, W.X. Why mercury concentration increases with fish size? Biokinetic explanation. Environmental pollution, v. 163, p. 192-198, 2012.

acerda, L.D.; Dias, F.J.; Marins, R.V.; Soares, T.M.; Godoy, J.M.O. \& Godoy, M.L.D. Pluriannual watershed discharges of $\mathrm{Hg}$ into a tropical semi-arid estuary of the Jaguaribe River, NE Brazil. Journal of the Brazilian Chemical Society, v. 24, n. 11, p. 1719-1731, 2013.

Moura, V.L. \& Lacerda, L.D. Contrasting Mercury Bioavailability in the Marine and Fluvial Dominated Areas of the Jaguaribe River Basin, Ceará, Brazil. Bulletin of environmental contamination and toxicology, p. 1-6, 2018. 
Pinho, A.P.; Guimarães, J.R.D.; Martins, A.S.; Costa, P.A.S.; Olavo, G. \& Valentin, J. Total mercury in muscle tissue of five shark species from Brazilian offshore waters: effects of feeding habit, sex, and length. Environmental Research, v. 89, n. 3, p. 250-258, 2002.

Reichmuth, J.M.; Weis, P. \& Weis, J.S. Bioaccumulation and depuration of metals in blue crabs (Callinectes sapidus Rathbun) from a contaminated and clean estuary. Environmental Pollution, v.158, n. 2, p. 361-368, 2010.

Rios, J.H.L.; Marins, R.V.; Oliveira, K.F.; \& Lacerda, L. D. Long-term (2002-2015) changes in mercury contamination in NE Brazil depicted by the mangrove oyster Crassostraea rhizophorae (Guilding, 1828). Bulletin of environmental contamination and toxicology, v. 97, n. 4, p. $474-479,2016$.

Soares, T.M.; Coutinho, D.A.; Lacerda, L.D.; Moraes, M.O. \& Rebelo, M.F. Mercury accumulation and metallothionein expression from aquafeeds by Litopenaeus vannamei Boone, 1931 under intensive aquaculture conditions. Brazilian Journal of Biology, v. 7, n.1, p. 131-137, 2011.

Soares, J.L.F.; Goch, Y.G.F.; Peleja, J.R.P.; Forsberg, B.R.; Lemos, E.J.S. \& Sousa, O.P. Bioacumulação de mercúrio total $(\mathrm{HgT})$ e hábitos alimentares de peixes da bacia do rio negro, Amazônia, Brasil.Biota Amazônia, v.6, n. 1, p. 102-106, 2016.

Streets, D.G.; Horowitz, H.M.; Jacob, D.J.; Lu, Z.; Levin, L.; Ter Schure, A.F. \& Sunderland, E.M. Total mercury released to the environment by human activities. Environmental Science and Technology, v. 51, n.11, p. 5969-5977, 2017.

USEPA. Guidance for Assessing Chemical Contaminant Data for Use in FishAdvisories. Vol. 1: Fish Sampling and Analysis. EPA 823-B-00-007. Office ofScience and Technology Office of Water U.S. Environmental Protection AgencyWashington, DC, v. 2, 2000. 